\section{DNA helical repeats}

SIR-The recent paper, "The helical repeat of double-stranded DNA varies as a function of catenation and supercoiling", by Wasserman et al. ${ }^{1}$ gives the misleading impression that the authors have solved the fundamental problem of the partition function between twist and writhe in supercoiled DNA. In fact, the authors did not measure the DNA helical repeat, $H$ (ref. 2), but a newly defined parameter, $h$ (ref. 3 ).

Whereas $H$ is uniquely determined by the three-dimensional structure of a given DNA molecule, $h$ measures the relationship between the DNA and a chosen reference surface ${ }^{3}$, and is therefore related to DNA structure only indirectly. Thus, the differences in $h$ calculated by Wasserman et al. correspond to changes in the relationship between the DNA and a reference surface rather than to changes in the internal structure of the DNA. The results of Wasserman et al. therefore have no relevance to the energetic constraints on DNA deformation. Furthermore, although the parameter $h$ has a clear biological meaning when it relates DNA to a real surface, its significance becomes doubtful when the surface is only imaginary.

ANDRZE STASIAK

Institute for Cell Biology,

Swiss Federal Institute of Technology, $\mathrm{CH}-8093$ Zurich, Switzerland

Claude Weber

Institute of Mathematics,

University of Geneva,

$\mathrm{CH}-1211$ Geneva, Switzerland

JACQUES DUBOCHET

Centre of Electron Microscopy,

University of Lausanne,

CH-1005 Lausanne, Switzerland

Cozzarelli et al. RePLy-For a DNA whose axis lies on a surface, real or virtual, there are two related measures of the turning of the double helix: twist (Tw), which measures a component of the rotation of one strand about the axis, and winding number $(\Phi)^{3}$, which is the sum of the periodic exposures of either strand away from the surface. The helical repeat of a DNA with $N$ base pairs has been defined in two different ways, namely $(N / \Phi)$ and $(N /$ Tw $)$ or $h$ and $H$, respectively, in the nomenclature of Stasiak et al. Both are valuable measures of DNA structure and differ only when DNA is supercoiled.

Contrary to the assertion of Stasiak et al. however, $h$ is often the property of biological significance. It is also much more easily measured. The parameter $h$ defines the sequence periodicity of nucleosomal DNA (ref. 4) and the phasing of protein-binding sites, and is, in fact, most commonly measured by the accessibility of DNA to chemical or enzymatic probes. In our experiments, $(N / \Phi)$ not
$(N / T w)$ is determined by the catenaneinduced supercoiling'. Supercoiling derived from catenation, linking number deficit, and the wrapping of DNA around histones, in all cases, leads to a change in $h$ (and also $H$ ); it is thus obviously incorrect for Stasiak et al. to assert that $h$ has no relevance to DNA structure or deformation. Stasiak et al. make an additional error; Wang ${ }^{2}$ did not distinguish $H$ from $h$ as he used relaxed DNA, for which the two are equal.

STEVEN A. WASSERMAN NiCHOLAS R. COZZARELLI

Department of Molecular Biology,

University of California,

Berkeley, California 94720, USA

Department of Mathematics, JAMES H. WHITE

University of California.

Los Angeles, California 90024, USA

1. Wasserman. S.A., White, J.H. \& Cozzarelli, N.R. Nature 334, 448-450 (1988).

2. Wang, J.C. Proc. natn. Acad. Sci. U.S.A. 76, 200-203 (1979).

3. White, J.H., Cozzarelli, N.R. \& Bauer, W.R. Science 241, 323-327 (1988)

4. Satchwell, S.C., Drew, H.R. \& Travers, A. J. molec. Biol. 191, 659-675 (1986)

\section{Cystic fibrosis}

SIR-Kitzis et al. ${ }^{1}$ confirm preliminary reports $^{2}$ of a segregation distortion of cystic fibrosis (CF) alleles with the sex of carriers. They use informative linked DNA markers to track CF alleles in families where there are carriers of the disease and count the numbers of carriers and non-carriers amongst unaffected siblings. The male/female ratio is as expected (1/1) and the carrier/non-carrier ratio follows mendelian expectation $(2 / 1)$. But the male/female ratio for $\mathrm{CF}$ carriers is $1.21 / 1.0$ (with a complementary $1.36 / 1$ female/male ratio for non-carriers). We bring to your attention work reported by Gedschold et ll $^{3}$, which we believe indirectly supports this segregation distortion phenomenon. These authors analysed questionnaires from a population-genetic study ${ }^{4}$, to show that for East German CF families, female carriers had more sibs (1.99 on average) than male carriers (1.66).

These two findings are remarkably consistent; the segregation distortion will generally lead to female carriers being found in larger sibships than male carriers. Using a male/female carrier ratio of 1.21 / 1.0 , the likelihood ratio of families ascertained through one female carrier with 1.99 unspecified sibs producing $n$ carrier sibs to families with one male carrier and 1.66 unspecified sibs producing $n$ carrier sibs is almost unity (that is, ignoring constant terms, $1.21 \times 1.66 / 1.0 \times 1.99=$ 1.0093)

Hence, we conclude that the segregation distortion of $\mathrm{CF}$ alleles with sex explains the differences in sibship sizes for male and female carriers. Linkage dis- equilibrium between the CF allele and alleles detected by DNA probes can be used to modify prior risks for individuals seeking carrier determination/exclusion ${ }^{5}$. Perhaps, now that the segregation distortion is confirmed, risk calculations should include the consultand's sex.

MARTIN FARRALL

Peter J. SCAMbler

\section{Department of Biochemistry and}

Molecular Genetics,

St Mary's Hospital Medical School,

Norfolk Place, London W2 1PG, UK

1. Kitzis, A. et al. Nature 336, 316 (1988).

2. Kitzis A et al Nature 333, 215 (1988)

3. Gedschold, J. et al. Hum. Genet. 80, 399-400 (1988)

4. Gedschold, J. Hum. Genet. 75, 277-280 (1987).

5. Farrall, M., Estivili, X. \&Williamson, R. Lancet ii, 156-157 (1987).

\section{Unhealthy genes}

SIR-In a recent review article (Nature 336, 435; 1988) Kondrashov asks whether future generations will gradually have less healthy genes. I found his article particularly interesting as I have recently started a debate in Norway concerning this problem.

The rate of mutation of human genes is no less than before. In industrialized societies, where the combination of medical care, lifestyles and reproduction strategies have decreased the selection for robust individuals, unwanted mutations will therefore be expected to accumulate.

The known genetic diseases represent only a small part of this problem, simply because they represent only a few specific mutations in a very limited number of genes. We have about 100,000 different genes (plus a large amount of DNA that influences gene activity) and each gene can mutate in many different ways. Some mutations are lethal, and therefore easily selected against, but most are probably either relatively neutral or slightly detrimental and do not cause any overt disease that can be traced to a specific mutation.

As a consequence we must, in the long run, expect an increased frequency of various health problems, implying that an increasing fraction of resources will need to be directed to health care.

I believe that an effort should be launched to evaluate the problem. The most important question is how fast the deterioration takes place. Depending on the outcome of this evaluation, it may be worth investigating possible means of dealing with the problem.

Kondrashov points out that the increase in unhealthy genes is a practically irreversible process. The irreversibility lies in the fact that we are dealing with humans and not animals, for which relevant selection programmes would be easy to implement. BJøRN GRINDE

National Institute of Public Health,

Geitmyrsveien 75 ,

0462 Oslo, Norway 